\title{
(1) The Effects of Massage Therapy on Pain Management in the Acute Care Setting
}

Rose Adams, MHA, BSW, LMT, ${ }^{1,2}$ Barb White, MS, LMT, , ${ }^{1,3,5}$ and Cynthia Beckett, PhD, RNC-OB, LCCE ${ }^{4,6}$

${ }^{1}$ Therapy Services, Massage Therapy; ${ }^{2}$ Member, Evidence-Based Practice Research Committee; ${ }^{3}$ Member, Ethics Committee; and ${ }^{4}$ Director, Pediatrics/Perinatal Services and Evidence-Based Practice, Flagstaff Medical Center, Flagstaff; ${ }^{5}$ Adjunct Faculty, Women's and Gender Studies, Northern Arizona University, Flagstaff; and ${ }^{6}$ Faculty Associate, College of Nursing and Healthcare Innovation, Arizona State University, Tempe, AZ, USA

Background: Pain management remains a critical issue for hospitals and is receiving the attention of hospital accreditation organizations. The acute care setting of the hospital provides an excellent opportunity for the integration of massage therapy for pain management into the team-centered approach of patient care.

Purpose and Setting: This preliminary study evaluated the effect of the use of massage therapy on inpatient pain levels in the acute care setting. The study was conducted at Flagstaff Medical Center in Flagstaff, Arizona - a nonprofit community hospital serving a large rural area of northern Arizona.

Method: A convenience sample was used to identify research participants. Pain levels before and after massage therapy were recorded using a $0-10$ visual analog scale. Quantitative and qualitative methods were used for analysis of this descriptive study.

Participants: Hospital inpatients $(n=53)$ from medical, surgical, and obstetrics units participated in the current research by each receiving one or more massage therapy sessions averaging 30 minutes each. The number of sessions received depended on the length of the hospital stay.

Result: Before massage, the mean pain level recorded by the patients was 5.18 [standard deviation (SD): 2.01]. After massage, the mean pain level was 2.33 (SD: 2.10). The observed reduction in pain was statistically significant: paired samples $t_{52}=12.43, r=.67, d=1.38, p<.001$. Qualitative data illustrated improvement in all areas, with the most significant areas of impact reported being overall pain level, emotional well-being, relaxation, and ability to sleep.

Conclusions: This study shows that integration of massage therapy into the acute care setting creates overall positive results in the patient's ability to deal with the challenging physical and psychological aspects of their health condition. The study demonstrated not only significant reduction in pain levels, but also the interrelatedness of pain, relaxation, sleep, emotions, recovery, and finally, the healing process.
KEYWORDS: Massage therapy, acute care, hospital, pain management, research, inpatients, patient care management, postoperative pain, anxiety, reflexology, craniosacral, acupressure, Swedish effleurage, pregnancy, cancer, fibromyalgia, relaxation

\section{INTRODUCTION}

Pain management within the acute care setting is a concern that is being carefully examined not only by individual hospitals, but also by accreditation organizations across the United States ${ }^{(1)}$. Massage therapy is one of the complementary and integrative medicine (CIM) therapies most often prescribed by physicians, and it is noted to be the most likely to be beneficial and the least likely to be harmful ${ }^{(2)}$. Studies have examined the experience of hospitalized patients and found that high levels of stress and anxiety can increase pain ${ }^{(3,4)}$ and slow a patient's recovery by limiting "physical functioning, including the ability to cough and breathe deeply, move, sleep, and perform self-care activities"'(5).

The Mayo Clinic of Rochester, Minnesota, conducted a systematic evaluation of the patient hospital experience and found that "tension, stress, pain, and anxiety were key challenges for patients"(6). The integration of massage therapy into the team approach in patient care constitutes a move forward that recognizes pain as the fifth vital sign after pulse, blood pressure, temperature, and respiratory rate ${ }^{(5)}$. Although each patient's healing process is unique ${ }^{(7)}$, common themes of healing recognized in the present study underlie the significance of a holistic approach to patient care.

Research has documented the use of massage therapy as an effective tool for pain management ${ }^{(8-10)}$, with the added benefit of producing few adverse reactions ${ }^{(11-13)}$. When, with cardiac surgery patients, opioid medications are initially necessary, the continued use of large doses can delay the recovery process and lead to prolonged hospitalization ${ }^{(11)}$. Patients with increased blood pressure because of stress may also benefit from massage therapy ${ }^{(14,15)}$. A study at the 
Mayo Clinic, in which 58 cardiac surgery patients postoperatively received $1-3$ massage therapy sessions of 20 minutes each, created evidence compelling enough for the Mayo Clinic to hire a full-time massage therapist to be available on the inpatient unit ${ }^{(6)}$.

Cardiac surgery patients often complain of back, shoulder, and neck pain from manipulation of the body during the surgical procedure and from physical manifestations of tension and stress ${ }^{(11)}$. When massage therapy is incorporated as part of the postsurgical protocol, fewer medications may be needed, providing an added advantage of fewer adverse side effects and acting as an effective adjunct or alternative to pharmaceuticals ${ }^{(10)}$

The gate-control theory of pain postulates that massage may be effective in "closing the gate" - that is, inhibiting the transmission of noxious stimuli by stimulating large nerve fibers that have been shown to alter pain perception ${ }^{(13)}$. In the acute care setting, health care professionals have a tendency to touch patients only when performing procedures, which can be uncomfortable and even painful. As White wrote, "Touch is often the most neglected or assaulted sense of the hospitalized patient"(16).

The relaxation response (RR) is the body's mechanism to decrease the level of psycho-physiologic arousal produced by stress ${ }^{(17)}$. Massage therapy can produce a RR that creates a calm state and enhances the ability to rest, qualities that are so essential for healing to occur ${ }^{(4)}$. In addition, the RR elicits physiological changes, including lower blood pressure and heart rate, decreased oxygen consumption and muscle tension, and lower levels of cortisol and noradrenaline ${ }^{(15)}$. "The majority of studies show that back massage induces a physiological or psychological relaxation response and that it is not injurious for critically ill patients with heart disease"(17).

Stressors experienced by hospital patients include excessive noise, lack of sleep, social isolation, enforced immobility, and pain from procedures. Anxiety and stress during cardiac catheterization can lengthen the hospital stay and increase the use of sedative medication before and during the procedure ${ }^{(3)}$. Hamel's research using a randomized clinical trial design with 46 participants demonstrated that a 20 -minute back massage successfully reduced blood pressure before cardiac catheterization ${ }^{(3)}$. Studies note that fear and anxiety are common emotions felt by cardiac surgery patients ${ }^{(6)}$, and as Moyer suggests, "There is much agreement that how a person feels, emotionally, is at least partly a function of that person's bodily state"(18). When patients have higher postoperative mobility, they may also have fewer serious postoperative complications, as demonstrated by Mitchinson and his colleagues in a randomized controlled trial of 605 veterans undergoing major surgery at Department of Veterans Affairs hospitals ${ }^{(5)}$.

Lack of sleep in the hospital environment is a well-known phenomenon and can delay a patient's recovery ${ }^{(4,9,17)}$. Hospital-induced sleep deprivation is generally remedied with medications ${ }^{(17)}$. Critically ill and elderly patients are a vulnerable population and may benefit from non-pharmacologic methods to promote sleep $^{(17)}$. By studying the amount of REM and NREM sleep in 69 elderly men, Richards found that sleep efficiency was $14.7 \%$ higher in patients who received a 6-minute back massage than in a control group ${ }^{(17)}$. That study is comparable with another that followed 30 patients with fibromyalgia who received 30 minutes of massage therapy twice weekly for 5 weeks. The patients experienced decreased depression, improved sleep (a greater number of sleep hours and fewer sleep movements) and decreased symptoms, including pain, fatigue, and stiffness ${ }^{(8)}$. Another study of 41 hospitalized oncology patients illustrated that sleep quality, pain, symptom distress, and anxiety all improved when massage therapy was given during the hospital stay ${ }^{(19)}$.

Egnew concludes that healing may be defined as "the personal experience of the transcendence of suffering"(7), and therefore each individual will have a personal concept of what "healing" means to them. Some aspects of healing are subjective and intensely personal, with different meanings for each person ${ }^{(7)}$. Integration of massage therapy may improve the healing environment for the patient, thus allowing the deeper aspects of psychological healing to occur along with physical healing.

As authors, we felt that it was important to include both quantitative and qualitative investigation. The value of including qualitative research is reflected by Kania and her colleagues in an article that describes how the use of the mixed methods approach "can provide highly valuable insights and a more complete understanding of the effectiveness of an intervention"(20). Using the mixed methods approach, the present study tests the research hypothesis "Does the use of massage therapy in an inpatient setting improve patient perception of pain management?"

\section{PARTICIPANTS AND METHODS}

This preliminary study enrolled a convenience sample of 65 inpatients admitted between October 1, 2006, and March 31, 2007, at a nonprofit community hospital serving a large rural area in the southwestern region of the United States. Criteria for inclusion in the study were a physician order for massage therapy, the patient's (or a family member's) ability to complete and sign a consent form, and willingness on the part of the patient to give feedback on the experience of hospital massage therapy and to return the qualitative survey after hospital discharge. Table 1 presents demographic data for the research participants.

The plan for the current study was submitted and approved by the hospital's institutional review board. Participation in the research project was voluntary. Informed consent, including confidentiality and the 
Table 1. Demographic Data of Research Participants

\begin{tabular}{lc}
\hline \multicolumn{1}{c}{ Characteristic } & Value \\
\hline Participants (n) & 65 \\
Mean age (years) & 45 \\
Sex [\% (women/men)] & $87 / 13$ \\
Ethnicity (\%) & \\
$\quad$ White & 82 \\
Native American & 12 \\
Hispanic & 2.3 \\
African American & 1 \\
Asian & 1 \\
Hospital unit (\%) & \\
Medical & 42 \\
Surgical & 31 \\
Obstetrics & 26 \\
\hline
\end{tabular}

right to withdraw from the study at any time, was obtained, and forms were completed before the initiation of any session for the research project. Participants were told that, whether they chose to partake or not to partake in the research, their regular treatment would continue unaffected. Standardization was assured by having each of the massage therapists use the same scripted dialogue when approaching potential subjects for the study. Visual analog scale (VAS) scores were obtained by the therapists before and after therapy. Participants were given the post-hospitalization survey at the end of their last session.

Three licensed massage therapists employed by the hospital provided the therapy sessions. The experience in massage therapy of the therapists (all female) ranged from 2 years to more than 20 years. Each had received additional training for working with hospitalized or medically frail patients, and all had worked in the acute care setting for 1 to 3 years.

Massage interventions consisted of 15-minute to 45-minute therapeutic massage sessions given at the bedside. Because of the disruptive nature of the hospital environment, the length of the sessions varied based on each participant's energy level and availability. Treatments included gentle Swedish effleurage or petrissage, acupressure, craniosacral therapy, or cross-fiber myotherapy with light-pressure effleurage and pressure points being the most commonly used modalities. The treatment area on the body varied according to participant need or concern, taking into consideration any contraindications, including but not limited to areas of acute injury and surgical and intravenous sites. Head, neck, shoulders, back, and feet were the areas most commonly chosen, with participants in either supine or side-lying positions. Participants were given a choice of unscented or lightly scented oils, and relaxing music was offered.

The survey used in this research project (Patient Survey for Massage Therapy Research) was adapted from a survey used by Motsinger in her Capstone Project, titled Development of an Inpatient Massage Therapy Program in an Allopathic Hospital. ${ }^{a}$ The survey asked about length of hospital stay, number of massages received, and whether massage therapy had improved, had had no effect, or had worsened the participant's overall pain levels, emotional well-being, ability to move, ability to participate in therapies, relaxation, ability to sleep, and recovery. Additionally, participants were asked if they felt that massage therapy had had an effect on their need for pain medication, how long the effects of the massage had lasted, and whether they planned to continue using massage therapy as part of their healing process. An open-ended inquiry at the end of the survey encouraged participants to comment freely about massage.

Quantitative and qualitative methods were used for analysis of this descriptive study. Demographic data, number of massage sessions, before-and-after pain levels using the VAS scale, survey data, and nursing comments were analyzed. Inferential statistical analysis was conducted using the paired $t$-test, with the significance level preset at $p<.05$.

Qualitative data drawn from nursing comments in a retrospective chart review and participant comments from the post-hospitalization survey were analyzed using the grounded theory method to code and label categories. "Grounded theory" can be described as a method of analysis that aims to develop middlerange theories from qualitative data. The founders of grounded theory, Glaser and Strauss, not only intended to conceptualize qualitative data, but also planned to demonstrate relationships between conceptual categories and to specify conditions within which theoretical relationships emerge ${ }^{(21)}$ (pp. 311-312). We used the grounded theory method to group qualitative responses into several categories to guide the analysis. These categories reflected responses by the participants and nurses regarding reactions to the massage therapy session or sessions the participant received while hospitalized. The themes generated demonstrate an interrelationship between categories and an overall theoretical sensitivity that supports the overriding theme that "massage therapy promotes recovery." Finally, all data were triangulated to determine whether massage therapy improves patient perception of pain management while in hospital.

\section{RESULTS}

\section{Quantitative Data}

From the initial sample of 65 participants, 53 completed the research project. Pain levels reported by the

a Motsinger S. Unpublished report for the Capstone Project for DPT. Flagstaff, AZ: Northern Arizona University; 2003. 
participants using the VAS ranged from $0-10$. The mean score before massage was 5.18 [standard deviation (SD): 2.01]. The mean score after massage was 2.33 (SD: 2.10). A comparison of pain levels before and after massage shows the individual responses by massage session (Fig. 1). The observed reduction in pain was statistically significant: paired samples $t_{52}=$ $12.43, r=.67, d=1.38, p<.001$ (Table 2).

The data show that most participants in the survey received 1 massage $(50.8 \%)$. Another $40 \%$ received $2-3$ massage sessions, and 6 participants $(9.2 \%)$ received more than 3 massage sessions. Most sessions $(83.9 \%)$ lasted 30 minutes, 14 sessions lasted 45 minutes $(14.9 \%)$, and 1 session lasted 15 minutes.

The effects of massage therapy were felt to last $1-4$ hours by 34 participants $(53.1 \%)$, and $4-$ 8 hours, by 13 participants (20.3\%). According to 9 participants $(14.1 \%)$, the effects lasted $8-24$ hours, and according to $7(10.9 \%)$, more than 24 hours. One

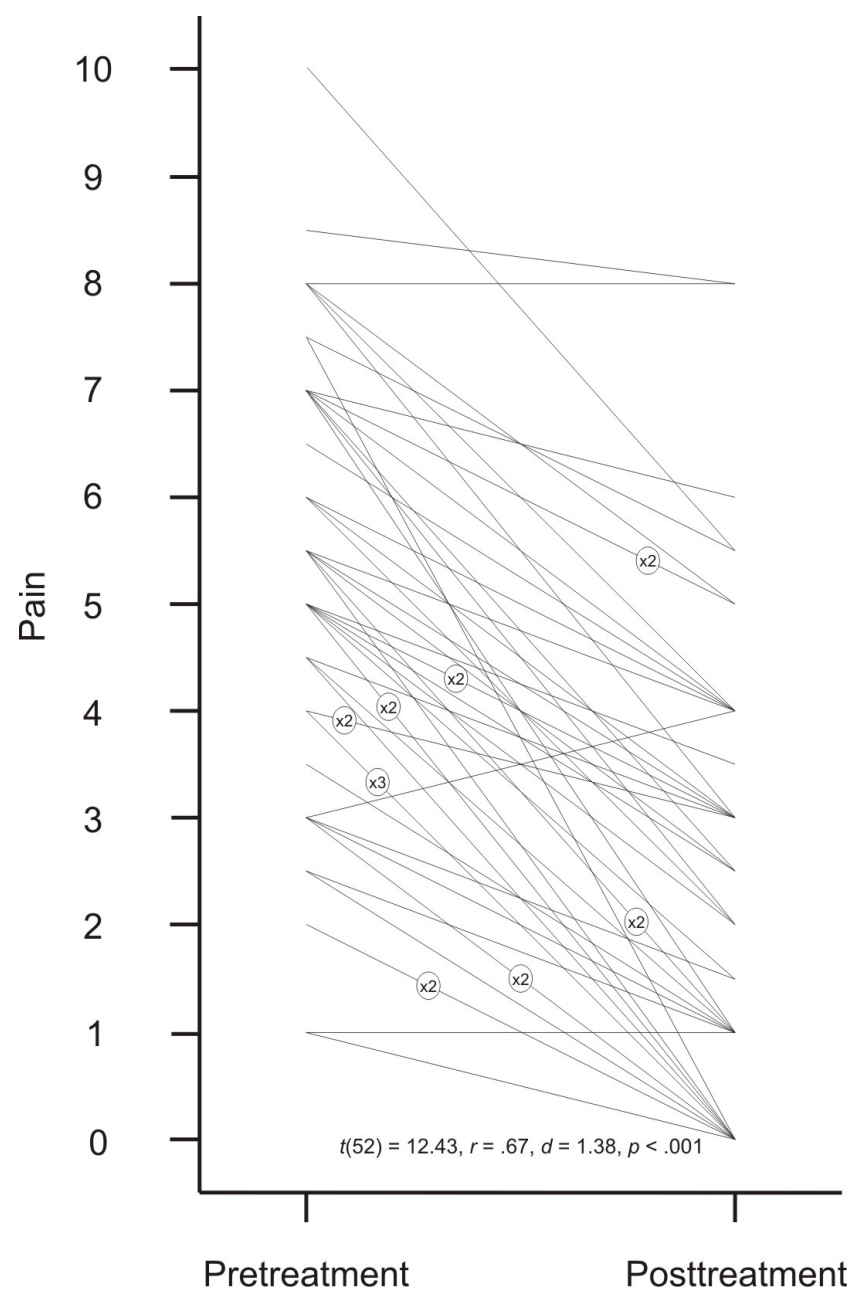

FIG. 1. Pain level on a $1-10$ visual analog scale before and after massage therapy in 65 inpatient research participants. Of the 65 charts reviewed, 53 charts contained complete data (before/after pain levels) and are shown here.
TABLE 2. Analysis of Pain Level Before and After Massage Therapy

\begin{tabular}{lc}
\hline \multicolumn{1}{c}{ Variable } & Value \\
\hline Mean pain score $( \pm \mathrm{SD})$ & \\
$\quad$ Before & $5.18(2.01)$ \\
After & $2.33(2.10)$ \\
Correlation & .67 \\
Standard mean error & 0.23 \\
$95 \%$ confidence limits & $(1.88,2.78)^{\mathrm{a}}$ \\
Significance (2-tailed) & $<.001^{\mathrm{a}}$ \\
Cohen's $d$ & 1.38
\end{tabular}

${ }^{a}$ Statistically significant.

$\mathrm{SD}=$ standard deviation

person felt no effect. The response to the question "Do you plan to continue using massage therapy in your healing process?" was yes in an impressive $67.2 \%$ of participants. Another 14.1\% responded no, and 18.8\% didn't know if they would continue with massage therapy after their hospitalization.

The survey reported participant perceptions concerning the effects of massage therapy on overall pain level, emotional well-being, ability to move, ability to participate in therapies, relaxation after massage, ability to sleep, contribution to faster recovery, and less need for pain medication after massage. Participants were asked if there was improvement, no change, or a worsening in the foregoing factors because of the massage. In all areas surveyed, a majority of participants reported an improvement, although some participants stated that they could not remember. Notably, no participant indicated a negative effect from massage therapy. The most significant areas of reported effect were overall pain level, emotional well-being, relaxation, and ability to sleep (Fig. 2).

Findings from the current preliminary study parallel existing research showing that pain levels significantly improved with a massage intervention as an adjunct to conventional treatments ${ }^{(5,19)}$. Other noteworthy observations included improved relaxation, emotional well-being, ability to sleep, and a reduction in the perception of use of pain medications.

\section{Qualitative Data}

Using the grounded theory method, "massage therapy promotes recovery" was the main theme identified. Within that theme, several subthemes emerged, including pain management (Table 3), ability to sleep, relaxation, emotional well-being, and healing. Each category is represented with comments from nurses or participants or both. Of the 65 participants in the study, $45(72.3 \%)$ returned surveys. At the end of the survey, an open-ended question encouraged participants to comment freely about 


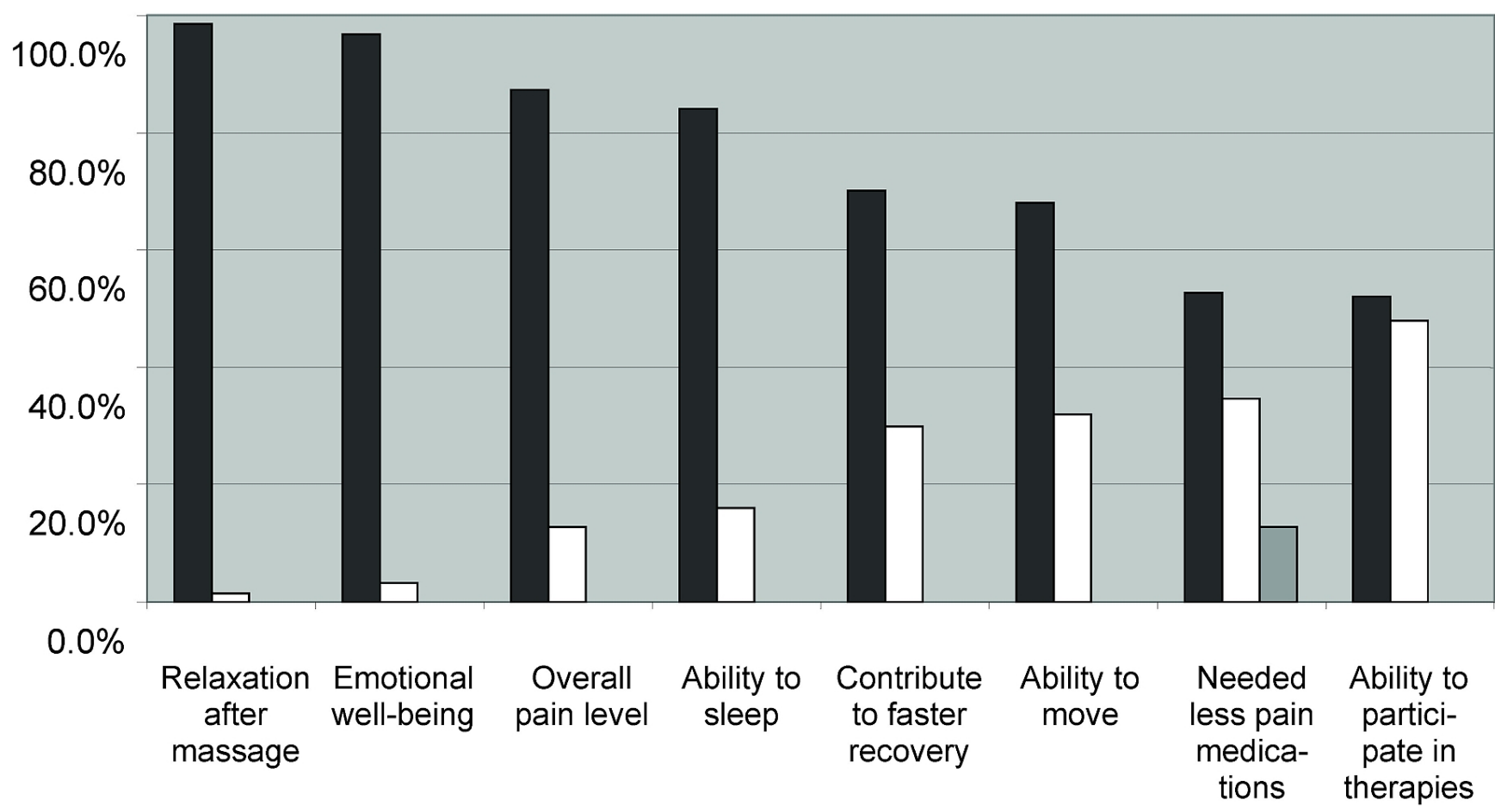

FIG. 2. Patient survey results. Black bars = improved; white bars $=$ no change; gray bar $=$ cannot remember.

TABLE 3. Qualitative Theme of Pain Management with Massage for Hospitalized Patients

$\begin{array}{ll}\text { Hospital inpatient } & \begin{array}{l}\text { "After three days in the hospital I was } \\ \text { suffering a migraine, nausea, and a lot of } \\ \text { body pain. After the massage my head- } \\ \text { ache was lessened and my body pain was } \\ \text { greatly reduced." } \\ \text { "Patient reports relaxation and pain relief } \\ \text { after massage-slept for three hours" } \\ \text { Nurse }\end{array} \\ \text { Cancer patient in } & \begin{array}{l}\text { "I looked forward to the massages I } \\ \text { received while in ICU-each helped to } \\ \text { ICU }\end{array} \\ & \text { reduce the pain." }\end{array}$

$\mathrm{ICU}=$ intensive care unit.

their experience of massage therapy. Qualitative responses were received from 33 participants.

In the medical charts of participants, 25 nursing comments relevant to the research project were found. All comments were categorized into themes and subthemes. Interrelating themes were also acknowledged.

\section{Pain Management}

Of the 33 qualitative responses from participants, 16 were related directly to pain management. Participants mentioned improved pain levels after surgery $(n=9)$, lessening of breast engorgement after a cesarean section $(n=1)$, decreased body and headache pain and intensity $(n=4)$, and decreased pain associated with cancer $(n=2)$. One cancer patient commented, "I looked forward to the massages I received while in ICU - each helped to reduce the pain." Another patient commented, "After three days in the hospital I was suffering a migraine, nausea, and a lot of body pain. After the massage my headache was lessened and my body pain was greatly reduced." While still hospitalized, one patient noted that "I've never had anything take this pain away completely." Still another noted that "I'm very much supportive of massage therapy as a healing and pain relief procedure." Not only was perception of pain lowered, but also perception of the need for pain medication. Significantly, more than half the participants $(52.7 \%)$ felt that they needed less pain medication after receiving massage therapy.

The responsibility of nurses for pain management plays a significant role in quality health care. Managing pain is a team effort between physicians, nurses, and other health care providers. Of the 25 comments by nursing staff, 16 referred to decreased pain levels or decreased necessity for pain medications after massage therapy. Nursing comments included "Patient states his neck pain lessened with massage," "Denies pain or needs ... had massage therapy this am, in no apparent distress," and "Massage therapy ordered and given with good relief."

\section{Sleep}

Comments about ability to sleep were often associated with pain relief. One patient noted that "massage brought dramatic pain relief and ability to sleep and an overall sense of well-being in a stressful 
environment." Another commented that "I fell asleep almost immediately after [the massage therapist] left." Nursing observations confirmed what patients stated on the survey. Nursing comments included "Patient reports relaxation and pain relief after massage - slept for three hours" and "Patient stated the massage was a big help in decreasing his pain and allowed him to relax enough to get a good nap today."

\section{Relaxation}

Relaxation can play a significant role in a patient's healing and recovery process. More than half the participants surveyed mentioned relaxation in their qualitative responses $(n=17)$. Patients mentioned relaxation, relief from muscle tension, and increased feelings of well-being and calm. Overall nervous tension and the stressful environment of the hospital were also mentioned. Remarks from patients relating to relaxation included "[massage therapy] was very helpful, soothing, comforting, and relaxing," and "this was very helpful to me, in that this is so pleasurable during an unpleasurable experience." A quadriplegic patient who received massage therapy commented that "overall well-being (emotional, physical, spiritual, patience, decreased anxiety, and decreased pain and spasticity) was improved immeasurably by massage therapy and subsequent relaxation."

In 10 comments from the nursing staff, the benefits of relaxation for their patients were mentioned. Nursing notes reflecting the benefits included "Patient seemed calmer tonight," "Patient reports improved muscle relaxation post massage therapy," and "Massage made her relaxed, resting comfortably, no distress."

\section{Emotional Well-being}

The interrelatedness of themes becomes apparent in how patient and nursing comments alike reflect the connection between emotional well-being and relaxation, pain relief, and ability to sleep. Participants mentioned emotional well-being 8 times in connection with decreased anxiety, state of mind, attitude improvement, and human contact. One patient commented "[massage therapy] was one of the few times I could look forward to human contact without the potential for pain (as opposed to shots, IV's ...)." Another patient described her experience with massage therapy this way: "I was so relieved and grateful. I was no longer crying and felt much better. I was so grateful for the body and mind relief." One of the participants in the research project was in advanced stages of cancer. Although she died, her husband returned the survey, commenting that his "wife appreciated the pain relief, and that "the massage brought a smile to her face'," also noting his own appreciation of the therapy.

Although nursing comments focused mainly on pain management and relaxation, 2 nursing notes articulated improved emotional well-being. A nurse in the Women's and Infant Center noted that "patient has been teary about infant in special care nursery, had a massage, now coping a little better." Another nurse commented "Patient seemed calmer tonight, not agitated, or hostile."

\section{Healing}

References to healing and subthemes of healing including spirituality, recovery, and therapeutic benefits were found in responses from 10 participants. One participant noted that "I feel massage is very important to helping patients heal, in so many ways," and another commented that "[massage therapy] was a very healing experience emotionally and physically." Other participant comments included "Reduction of stress also was very helpful in recovery," "It was very therapeutic," and "It is such a healing process and definitely relaxing." No nursing comments on the theme of healing were found.

\section{DISCUSSION}

The experience of hospitalization creates pain and anxiety for many people, regardless of their underlying medical condition. The goal of the present study was to explore how massage therapy would affect a patient's perception of pain in the acute care setting. Previous studies have established the benefits of massage therapy for patients suffering from particular illnesses - for example, cancer ${ }^{(19)}$ - and cardiac surgery or procedures ${ }^{(3,6,17)}$. Other studies have focused on patient experiences within particular hospital units including transplantation, neuroscience, and rehabilitation ${ }^{(4)}$. By selecting research participants in units throughout the hospital, with a wide variety of diagnoses and reasons for hospitalization, our project provides a unique picture of how massage therapy may benefit any patient coping with the pain and stress associated with hospitalization, offering a strong argument that massage is an effective adjunct therapy for pain management.

The primary findings of this preliminary study show a strong correlation between reduction of pain levels after massage therapy and statistically significant differences in pain scores before and after massage. The perception among participants of improved pain levels and less need for pain medication underscore the promise of massage therapy's positive effect on pain management protocols. In addition, a majority of patients felt that massage therapy contributed to increased relaxation, emotional well-being, ability to sleep, ability to move and to participate in other therapies, and faster recovery. For most patients, the effects of the session lasted $1-4$ hours, with some participants experiencing benefits for more than 24 hours.

Reports of improved levels of relaxation after massage therapy were received from $98 \%$ of the research 
study participants. The fact that patients throughout the various hospital units, with a wide variety of pre-massage pain levels, experienced relaxation through massage therapy indicates the true potential for massage to support healing for hospitalized patients. This finding was reiterated in comments from patients and nurses alike. By accessing a patient's ability to relax, massage therapy addresses a variety of needs. Indeed, the RR may be the most profound mechanism through which massage therapy helps the hospitalized patient.

In addition to relaxation, massage therapy also counters another fundamental aspect of hospitalization, the sense of isolation experienced by many patients. In the present research study, participants reported an improvement in emotional well-being - an aspect of healing that may speak to the need for human touch. More and more hospitals are recognizing the importance of touch for the hospitalized patient ${ }^{(4,6)}$. As the face of health care changes in the coming years, it is a hopeful sign that safe, skillful touch is being recognized as a mechanism of healing for patients in the acute care setting.

Participation in our research study was limited to adults whose medical circumstances allowed them to receive massage therapy and to complete the study paperwork. The study does not reflect the perceptions of patients whose energy or pain levels precluded them from participation. Patients whose level of pain did not allow for participation may have found less benefit from massage therapy, revealing the need for fully integrative services in which massage is merely one component of a comprehensive pain management protocol.

Another limitation of the present study is the lack of collection of physiological data, including heart rate, blood pressure, and oxygen levels. The absence of data on the physiological indicators of pain and the RR means that the study relied on participant perceptions without additional external measures to verify participant responses to massage therapy. Pain is an inherently subjective experience that includes physical and emotional elements. Within the hospital environment, health care workers rely on patient perceptions for pain management. The present study thus reflects current standards for assessing the effectiveness of various interventions to address pain in patients.

The current project, designed to gather preliminary data on the research hypothesis, did not use a control group. The selection of additional patients in units throughout the hospital, combined with randomization to groups, would have required substantial additional resources. However, future studies on massage therapy in an acute care setting may benefit from the addition of a control group. Such research may also help to identify specific types of massage therapy that are most effective in the acute care setting.

\section{CONCLUSIONS}

Evidence-based research continues to confirm the importance of human touch to balance the high technology of today's health care practices. The further integration of CIM therapies such as massage into the hospital offers the possibility to improve the experience for patients who face physical, psychological, and social challenges in an unfamiliar environment. A large and growing body of research, including the current project, justifies the use of massage therapy for pain management in the acute care setting. Massage therapy can provide pain relief and relaxation, can support a patient's emotional well-being and recovery, and can ultimately aid in the healing process for hospitalized patients.

\section{ACKNOWLEDGMENTS}

This research was conducted at Flagstaff Medical Center, Flagstaff, Arizona, from October 2006 to March 2007.

The authors thank Alisha Witcomb, LMT, for her contribution as one of the three massage therapists involved in this study and as an integral collaborator in the data collection process. The authors also express their gratitude to Lori Pearlmutter, PT, MPH. Lori was inspirational and supportive throughout the process, as a co-investigator and a mentor.

\section{CONFLICT OF INTEREST NOTIFICATION}

The authors declare that no conflicts of interest are associated with this research project or publication of findings.

\section{COPYRIGHT}

Published under the CreativeCommons Attribution-NonCommercial-NoDerivs 3.0 License.

\section{REFERENCES}

1. Pearson Education. American Academy of Pain Management accreditation helps pain organizations meet JCAHO standards. Pearson Education, Bridging the Gap website. http://test.pearsonassessments.com/bridginggap/fall2001-p1.htm. Published Fall 2001. Updated n.d. Accessed July 12, 2007.

2. Ezzo J. What can be learned from Cochrane systematic reviews of massage that can guide future research? J Altern Complement Med 2007; 13(2): 291-295.

3. McCaffrey R, Taylor N. Effective anxiety treatment prior to diagnostic cardiac catheterization. Holist Nurs Pract 2005; 19(2): 70-73. 
4. Smith MC, Stallings MA, Mariner S, Burrall M. Benefits of massage therapy for hospitalized patients: a descriptive and qualitative evaluation. Altern Ther Health Med 1999; 5(4): 64-71.

5. Mitchinson AR, Kim HM, Rosenberg JM, Geisser M, Kirsh M, Cikrit D, et al. Acute postoperative pain management using massage as an adjuvant therapy: a randomized trial. Arch Surg 2007; 142(12): 1158-1167.

6. Cutshall SM, Fenske LL, Kelly RF, Phillips BR, Sundt TM, Bauer BA. Creation of a healing enhancement program at an academic medical center. Complement Ther Clin Pract 2007; 13(4): 217-223.

7. Egnew TR. The meaning of healing: transcending suffering. Ann Family Med 2005; 3(3): 255-262.

8. Field T, Diego M, Cullen C, Hernandez-Reif M, Sunshine W, Douglas S. Fibromyalgia pain and substance P decrease and sleep improves after massage therapy. J Clin Rheumatol 2002; 8(2): 72-76.

9. Hernandez-Reif M, Field T, Krasnegor J, Theakston H. Lower back pain is reduced and range of motion increased after massage therapy. Int J Neurosci 2001; 106(3-4): 131-145.

10. Melancon B, Miller LH. Massage therapy versus traditional therapy for low back pain relief: implications for holistic nursing practice. Holist Nurs Pract 2005; 19(3): 116-121.

11. Anderson PG, Cutshall SM. Massage therapy: a comfort intervention for cardiac surgery patients. Clin Nurse Spec 2007; 21(3): 161-165.

12. Cassileth B, Trevisan C, Gubili J. Complementary therapies for cancer pain. Curr Pain Headache Rep 2007; 11(4): 265-269.

13. Ferrell-Torry AT, Glick OJ. The use of therapeutic massage as a nursing intervention to modify anxiety and the perception of cancer pain. Cancer Nurs 1993; 16(2): 93-101.
14. Aourell M, Skoog M, Carleson J. Effects of Swedish massage on blood pressure. Complement Ther Clin Pract 2005; 11(4): 242-246.

15. Benson H. The Relaxation Response. New York, NY: William Morrow and Company; 1975: 99-110.

16. White JA. Touching with intent: therapeutic massage. Holist Nurs Prac 1988; 2(3): 63-67.

17. Richards KC. Effect of a back massage and relaxation intervention on sleep in critically ill patients. Am J Crit Care 1998; 7(4): 288-299.

18. Moyer CA. Affective massage therapy. Int $J$ Ther Massage Bodyw 2009; 1(2): 4.

19. Smith MC, Kemp J, Hemphill L, Vojir CP. Outcomes of therapeutic massage for hospitalized cancer patients. $J$ Nurs Scholarsh 2002; 34(3): 257-262.

20. Kania A, Porcino A, Vehoef MJ. Value of qualitative research in the study of massage therapy. Int J Ther Massage Bodyw 2008; 1(2): 6-10.

21. Charmaz K. Qualitative interviewing and grounded theory analysis. In: Holstein A, Gubrium JF, eds. Inside Interviewing : New Lenses, New Concerns. Thousand Oaks, CA: Sage Publications; 2003: 311-30.

Corresponding author: Rose Adams, Flagstaff Medical Center, Therapy Services, Massage Therapy, 1215 N. Beaver Street, Flagstaff, Arizona 86001 U.S.A.

E-mail:rose.adams@nahealth.com 